\title{
A Novel Approach for Spectral Efficiency Analysis in MIMO Cellular Networks
}

\author{
Geordie George, Ratheesh K. Mungara and Angel Lozano \\ Universitat Pompeu Fabra (UPF) \\ 08018 Barcelona, Spain. \\ Email:\{geordie.george, ratheesh.mungara, angel.lozano\}@upf.edu
}

\author{
Martin Haenggi \\ University of Notre Dame \\ Notre Dame, IN 46556, USA. \\ Email: mhaenggi@nd.edu
}

\begin{abstract}
The application of stochastic geometry to the analysis of wireless networks is shown to be propelled by $(i)$ a clean separation of time scales, (ii) abstraction of small-scale effects via ergodicity, and (iii) an interference model reflecting the receiver's lack of knowledge of how individual interference terms are faded. These steps render the analysis simpler and more precise, and more amenable to incorporating subsequent features. Specifically, the paper presents easy-to-evaluate expressions for the ergodic spectral efficiency of cellular networks with single-user multipleinput multiple-output (MIMO).
\end{abstract}

\section{INTRODUCTION}

Although the stochastic modelling of wireless networks would seem mostly amenable to those devoid of fixed infrastructure, i.e., ad hoc networks, Andrews et al. [1] proved the truly remarkable effectiveness of stochastic modelling also in cellular networks — even with simple Poisson point processes (PPPs). Indeed, while more sophisticated spatial distributions could appear to better capture the relative regularity of actual base station (BS) placements, PPPs lead-because of shadowing - to remarkably precise characterizations of signal strengths and interference. In fact, PPP-based characterizations represent the limit to which actual behaviors converge as the shadowing strengthens [2], [3].

We target the ergodic spectral efficiency of Poisson cellular networks, but differently from previous works. An alternative approach to model interference takes us on a route that proves greatly advantageous because it yields solutions that are simpler and more precise, and because it opens the door to including further aspects such as MIMO (included here) and cell sectorization (presented in an extended version of this paper [4]). The modeling approach that unlocks these new analytical possibilities is not arbitrary, but rather based on sound arguments:

1) A clean separation between large- and small-scale channel features, recognizing that the phenomena that give rise to these features are distinct.

2) A decided embrace of ergodic performance metrics with respect to small-scale features, reflecting that such smallscale ergodicity captures well the operating conditions of modern wireless systems [5], [6].

This work was supported by Project TEC2015-66228-P (MINECO/FEDER, UE) and by the U.S. NSF through award CCF 1525904.
3) The admission that each receiver can track the fading of its intended signal, but not the fading of individual interference terms.

With small- and large-scale features decoupled, ergodicity enables abstracting out the former so as to focus the stochastic geometry thrust on the latter. This enables advancing the analysis on all fronts: simplicity, accuracy, and generality.

\section{Network Modelling}

\section{A. Separation of Scales}

The separation of large-scale propagation phenomena (distance-dependent path loss and shadowing) and small-scale multipath fading has long been instrumental in the study of wireless networks, enabling characterizations that would otherwise be unwieldy. Over the span of each signal codeword, while the large-scale features can be regarded as constant, the small-scale fading may or may not remain constant, depending on the fading coherence and on how codewords are arranged in time/frequency. This dichotomy leads to two classic information-theoretic channel idealizations:

- Nonergodic. Fading random, but fixed over the codeword.

- Ergodic. Fading random and exhibiting sufficient variability over the codeword to essentially reveal its distribution.

These two idealizations map, respectively, to outage and ergodic definitions for the spectral efficiency. The ergodic definition is the most representative for modern systems where codewords can be interspersed over wide bandwidths, across hybrid-ARQ repetitions, and possibly on multiple antennas, while being subject to scheduling and link adaptation. As argued in [6], the balance of these mechanisms is indeed best abstracted by ergodic spectral efficiencies involving expectations over the small-scale fading, with the large-scale features held fixed. It is at this point that stochastic geometry should enter the analysis, when the small-scale effects have been abstracted out and we can zoom out to cleanly examine the large-scale ones.

\section{B. Large-scale Modeling}

Consider the downlink of a cellular network with omnidirectional antennas, where the signals are subject to path loss with exponent $\eta>2$ as well as shadowing.

It has been recently shown [2], [3] that, regardless of the BS positions (under only a very mild homogeneity condition), an 
increasing shadowing standard deviation renders the network progressively PPP-like from the vantage of any given user, making the powers that a user receives from any population of BSs look as if they originated from PPP-distributed BSs. This justifies adopting a PPP model for the spatial distribution of BSs, say a process $\Phi_{\mathrm{b}} \subset \mathbb{R}^{2}$, without the need to explicitly model the shadowing as it is already implicitly captured by the PPP nature of the network.

As of the spatial distribution of users, a good starting point is an independent PPP $\Phi_{\mathrm{u}}$ with density $\lambda_{\mathrm{u}}$.

Without loss of generality, we conduct the analysis from the perspective of a user at the origin, which becomes the typical user under expectation over $\Phi_{\mathrm{b}}$. Denoting by $r_{k}$ the distance between such user and the $k$ th BS, we index the BSs in increasing order of $r_{k}$, i.e., such that $r_{k}<r_{k+1}$ for $k \in$ $\mathbb{N}_{0}$. Since, in terms of $\Phi_{\mathrm{b}}$, the only large-scale propagation mechanism is path loss, the user at the origin receives the strongest power from the $\mathrm{BS}$ at $r_{0}$, which we deem the serving BS.

\section{Small-scale Modeling}

Let with BSs and users having a single antenna for now, and let each receiver know the fading of only its intended signal. Denoting by $P$ the power measured at $1 \mathrm{~m}$ from a BS transmitter, at symbol $n$ the user at the origin observes

$$
y[n]=\sqrt{P r_{0}^{-\eta}} H_{0}[n] s_{0}[n]+z[n]
$$

where the leading term is the signal from the serving BS while

$$
z[n]=\sum_{k=1}^{\infty} \sqrt{P r_{k}^{-\eta}} H_{k}[n] s_{k}[n]+v[n]
$$

is the interference from all other BSs, plus thermal noise $v$. In turn, $s_{k}$ is the signal transmitted by the $k$ th BS and $H_{k}$ is the corresponding fading coefficient. The fading coefficients $\left\{H_{k}\right\}_{k=0}^{\infty}$ are independent and of unit power, but otherwise arbitrarily distributed, while $v \sim \mathcal{N}_{\mathbb{C}}\left(0, N_{0}\right)$. The signal is distributed as $s_{k} \sim \mathcal{N}_{\mathbb{C}}(0,1)$, a choice that is justified later.

Conditioned on $\left\{r_{k}\right\}_{k=0}^{\infty}$, which are fixed as far as the small-scale modeling is concerned, the instantaneous SINR at symbol $n$ is

$$
\operatorname{SINR}[n]=\frac{P r_{0}^{-\eta}\left|H_{0}[n]\right|^{2}}{P \sum_{k=1}^{\infty} r_{k}^{-\eta}\left|H_{k}[n]\right|^{2}+N_{0}} .
$$

\section{INTERFERENCE MODELING}

With $H_{0}[1], \ldots, H_{0}[N]$ known by the receiver at the origin, the mutual information (in bits/symbol) over codewords spanning $N$ symbols is

$\frac{1}{N} I\left(s_{0}[1], \ldots, s_{0}[N] ; y[1], \ldots, y[N] \mid H_{0}[1], \ldots, H_{0}[N],\left\{r_{k}\right\}\right)$

which, with IID codeword symbols, becomes

$$
\frac{1}{N} \sum_{n=1}^{N} I\left(s_{0}[n] ; y[n] \mid H_{0}[n],\left\{r_{k}\right\}_{k=0}^{\infty}\right) .
$$

As argued, codewords are nowadays long enough - thousands of symbols-and arranged in such a way-interspersed in time, frequency, and increasingly across antennas-so as to experience sufficiently many fading swings for an effective averaging of the mutual information to take place over the small-scale fading. From the stationarity and ergodicity of such fading over the codeword, the averaging in (4) becomes an expectation over $H_{0}$ and confers the significance of the ergodic spectral efficiency (in bits/s/Hz)

$$
\begin{aligned}
C_{\text {exact }} & =\mathbb{E}_{H_{0}}\left[I\left(s_{0} ; y \mid H_{0},\left\{r_{k}\right\}_{k=0}^{\infty}\right)\right] \\
& =\mathbb{E}_{H_{0}}\left[I\left(s_{0} ; \sqrt{P r_{0}^{-\eta}} H_{0} s_{0}+z \mid H_{0},\left\{r_{k}\right\}_{k=0}^{\infty}\right)\right] .
\end{aligned}
$$

This quantity, a baseline in the sequel, does not admit explicit expressions. Rather, its evaluation requires computationally intensive Monte-Carlo simulation [4, App. A] [7, App. A] and a 64-core high-performance computing cluster is employed to generate the corresponding results throughout this paper; for all such results, $99 \%$ confidence intervals are provided.

Let us examine the local distribution, for some given $\left\{r_{k}\right\}_{k=1}^{\infty}$, of the interference-plus-noise $z$ in (2). The first thing to note is that, without further conditioning on $\left\{H_{k}\right\}_{k=1}^{\infty}$, i.e., without the receiver knowing the fading coefficients from all interfering BSs, the local distribution of $z$ is generally not Gaussian. Conditioned only on $\left\{r_{k}\right\}_{k=1}^{\infty}$, the distribution of $z$ is actually highly involved; in Rayleigh fading, for instance, it involves products of Gaussians. While irrelevant to the localaverage SINR, since only the variance of $z$ matters in that respect, the non-Gaussianity of $z$ is relevant to the spectral efficiency, which does depend on the distribution of $z$.

It is nevertheless customary to analyze $C_{\text {exact }}$ in the form it would have if $\left\{H_{k}\right\}_{k=1}^{\infty}$ were actually known by the receiver at the origin and $z$ were consequently Gaussian, namely

$$
C_{\mathrm{ub}}=\mathbb{E}_{\left\{H_{k}\right\}_{k=0}^{\infty}}\left[\log _{2}\left(1+\frac{P r_{0}^{-\eta}\left|H_{0}\right|^{2}}{P \sum_{k=1}^{\infty} r_{k}^{-\eta}\left|H_{k}\right|^{2}+N_{0}}\right)\right]
$$

where the tacit redefinition of $z$ as Gaussian is unmistakable from $I\left(s_{0} ; \sqrt{\gamma} s_{0}+z\right)=\log _{2}(1+\gamma)$, which holds only when $s_{0}$ and $z$ are Gaussian. As it turns out, a Gaussian modeling of $z$ is not unreasonable because, if a decoder is intended for Gaussian interference-plus-noise (either by design or because the distribution thereof is unknown), then the spectral efficiency is precisely as if the interference-plus-noise were indeed Gaussian, regardless of its actual distribution [8]. And, once $z$ is taken to be Gaussian, the capacity-achieving signal distribution is also Gaussian, validating our choice for $s_{0}$. At the same time, the granting of $\left\{H_{k}\right\}_{k=1}^{\infty}$ as additional side information to the receiver renders (7) an upper bound to $C_{\text {exact }}$, hence the denomination $C_{\mathrm{ub}}$.

While much more tractable than $C_{\text {exact }}$, the form of $C_{\mathrm{ub}}$ has the issue of depending not only on $H_{0}$, but further on $\left\{H_{k}\right\}_{k=1}^{\infty}$. This still clutters its analysis considerably.

Alternatively, what we propound in this paper is to model $z$ as Gaussian, but forgoing the small-scale variations in its 
power, i.e., to use

$$
z \sim \mathcal{N}_{\mathbb{C}}\left(0, P \sum_{k=1}^{\infty} r_{k}^{-\eta}+N_{0}\right)
$$

This model has the virtue of rendering $z$ Gaussian without the strain of gifting the receiver with $\left\{H_{k}\right\}_{k=1}^{\infty}$. The closeness between the distribution of $z$ as originally defined in (2) and the distribution in (8) has been tightly bounded by means of the Kolmogorov-Smirnov distance [9]. With $z$ as in (8), the instantaneous SINR then becomes

$$
\operatorname{SINR}=\frac{P r_{0}^{-\eta}\left|H_{0}\right|^{2}}{P \sum_{k=1}^{\infty} r_{k}^{-\eta}+N_{0}},
$$

and the corresponding ergodic spectral efficiency is

$$
C=\mathbb{E}_{H_{0}}\left[\log _{2}\left(1+\frac{P r_{0}^{-\eta}\left|H_{0}\right|^{2}}{P \sum_{k=1}^{\infty} r_{k}^{-\eta}+N_{0}}\right)\right]
$$

which is the quantity we work with henceforth.

Since, with Gaussian codewords and a given variance for $z$, the mutual information is minimized when $z$ is Gaussian [10], we have that $C \leq C_{\text {exact }}$. The similarity between $C$, characterized analytically, and $C_{\text {exact }}$, obtained through MonteCarlo simulation, is illustrated throughout the paper.

Contrasting the instantaneous SINR expressions in (3) and (9), the analytical virtues of our model for $z$ become evident once we rewrite the latter as $\operatorname{SINR}=\rho\left|H_{0}\right|^{2}$, where

$$
\rho=\frac{P r_{0}^{-\eta}}{P \sum_{k=1}^{\infty} r_{k}^{-\eta}+N_{0}}
$$

is the local-average ${ }^{1}$ SINR at the origin, fixed over any entire codeword and cleanly separated from the fluctuant term $\left|H_{0}\right|^{2}$; this reflects the decoupling between the large- and small-scale dependences. In interference-limited conditions $\left(P / N_{0} \rightarrow \infty\right)$, the local-average SINR specializes to $\rho=r_{0}^{-\eta} / \sum_{k=1}^{\infty} r_{k}^{-\eta}$. For given BS and user locations, $\rho$ becomes determined and the conditional distribution of the instantaneous SINR is then given directly by that of $\left|H_{0}\right|^{2}$, i.e., by the $\mathrm{CDF} F_{\left|H_{0}\right|^{2}}(\cdot)$, while the ergodic spectral efficiency of a user with localaverage $\operatorname{SINR} \rho$ is

$$
\begin{aligned}
C(\rho) & =\mathbb{E}_{H_{0}}\left[\log _{2}\left(1+\rho\left|H_{0}\right|^{2}\right)\right] \\
& =\int_{0}^{\infty} \log _{2}(1+\rho \xi) \mathrm{d} F_{\left|H_{0}\right|^{2}}(\xi)
\end{aligned}
$$

which, through $\rho$, sets the stage for further computations involving the geometry of the network.

Example 1. In Rayleigh fading, $F_{\left|H_{0}\right|^{2}}(\xi)=1-e^{-\xi}$, from which

$$
C(\rho)=e^{1 / \rho} E_{1}\left(\frac{1}{\rho}\right) \log _{2} e,
$$

where $E_{n}(x)=\int_{1}^{\infty} t^{-n} e^{-x t} \mathrm{~d} t$ is an exponential integral.

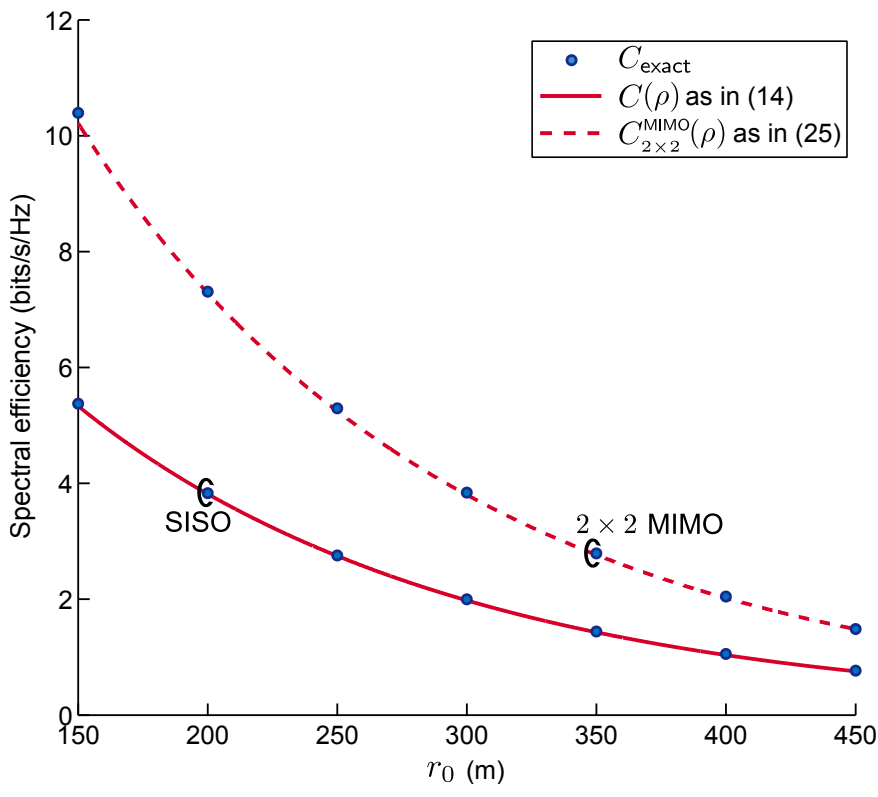

Fig. 1: Spectral efficiency vs. $r_{0}$ for $\lambda_{\mathrm{b}}=2 \mathrm{BSs} / \mathrm{km}^{2}$ and $\eta=4$. The $99 \%$ confidence intervals around $C_{\text {exact }}$ range from \pm 0.029 at $r_{0}=150 \mathrm{~m}$ to \pm 0.009 at $r_{0}=450 \mathrm{~m}$, for both SISO and MIMO.

For fading distributions other than Rayleigh, or with MIMO or other features, corresponding forms can be obtained for $C(\rho)$, always with the key property of these being a function of $\rho$ and not of the instantaneous fading coefficients.

Example 2. Let us apply (14) to a network with 100 interfering BSs. To typify the network, we set $r_{k}$ to the expected value of the distance to the $k$ th nearest point in a PPP: $r_{k}=\frac{\Gamma(k+1.5)}{\sqrt{\pi \lambda_{\mathrm{b}}} \Gamma(k+1)}$ for $k=1, \ldots, 100$ [11]. We further set $\eta=4$ and $\lambda_{\mathrm{b}}=2 \mathrm{BSs} / \mathrm{km}^{2}$ and neglect the noise. Shown in Fig. 1 is $C(\rho)$ in (14) compared against $C_{\text {exact }}$. The same comparison is provided for MIMO with two transmit and two receive antennas, whose ergodic spectral efficiency $C_{2 \times 2}^{\text {мІмо }}(\cdot)$ is derived later in (25). In both cases the differences are minute, supporting our interference modeling approach.

\section{LOCAL-AVERAGE SINR: Distribution}

Thus far, the link distances $\left\{r_{k}\right\}$ have been conditioned upon. Once they are released, $\rho$ becomes itself a random variable whose distribution is induced by the process $\Phi_{\mathrm{b}}$. The corresponding $\mathrm{CDF}, F_{\rho}(\cdot)$, is to be a central ingredient in our analysis of the ergodic spectral efficiency. This important function, extensively utilized by system designers, has traditionally been obtained by means of simulation over lattice networks with shadowing [12].

Here we analytically characterize $F_{\rho}(\cdot)$ for Poisson networks, implicitly incorporating shadowing, and specifically in interference-limited conditions. This distribution was established in [13]-[16], very compactly for arguments above 1

\footnotetext{
${ }^{1}$ The term "local-average" indicates averaging over the small-scale fading only. It follows that the "local-average SINR" is its average value over a region small enough for the path loss (and the shadowing, if applicable) not to change noticeably.
} 
TABLE I: Parameter $s^{\star}$ and the corresponding $\delta$ for typical values of the path loss exponent $\eta$.

\begin{tabular}{|c|c|c|c|c|c|}
\hline$\eta$ & $\delta$ & $s^{\star}$ & $\eta$ & $\delta$ & $s^{\star}$ \\
\hline \hline 3.5 & 0.571 & -0.672 \\
\hline 3.6 & 0.556 & -0.71 \\
\hline 3.7 & 0.540 & -0.747 \\
\hline 3.8 & 0.526 & -0.783 & 0.513 & -0.819 \\
\hline 4 & 0.5 & -0.854 \\
\hline
\end{tabular} \begin{tabular}{|c|c|c|}
\hline 4.1 & 0.488 & -0.888 \\
\hline
\end{tabular}

and in a still manageable form between $1 / 2$ and 1 , but in an accelerating cumbersome fashion (involving progressively higher-dimensional integrations) as the argument dips below $1 / 2$ [15, Sec. V.A], [16, Cor. 19]. As alternatives, [17] derived $F_{1 / \rho}(\cdot)$ in the Laplace domain, which would then require numerical inversion, while [18] showed that the lower tail $(\theta \rightarrow 0)$ of $F_{\rho}(\theta)$ satisfies

$$
\log F_{\rho}(\theta)=\frac{s^{\star}}{\theta}+o(1)
$$

with $s^{\star}<0$ being the solution to

$$
s^{\star \delta} \bar{\Gamma}\left(-\delta, s^{\star}\right)=0
$$

where $\bar{\Gamma}$ is the lower incomplete gamma function. For compactness, we have introduced the shorthand notation $\delta=2 / \eta$. Since it only depends-through $\delta$-on the path loss exponent $\eta$, the parameter $s^{\star}$ can be precomputed for all relevant values thereof. Values of $s^{\star}$ for typical path loss exponents are listed in Table I.

The approach we take is to apply the exact form given in [15, Sec. V.A], [16, Cor. 19] down to $\theta=1 / 2$ and then (15) for $\theta<1 / 2$. This combination gives

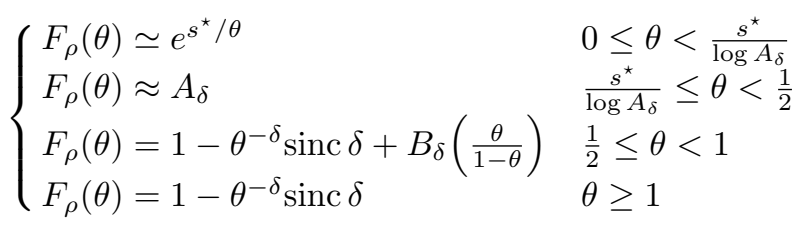

where " $\simeq "$ indicates asymptotic $(\theta \rightarrow 0)$ equality while

$$
A_{\delta}=1-2^{\delta} \operatorname{sinc} \delta+B_{\delta}(1)
$$

and

$$
B_{\delta}(x)=\frac{\delta \operatorname{sinc}^{2}(\delta) \Gamma^{2}(\delta+1){ }_{2} F_{1}(1, \delta+1 ; 2 \delta+2 ;-1 / x)}{x^{1+2} \delta \Gamma(2 \delta+2)}
$$

with ${ }_{2} F_{1}$ the Gauss hypergeometric function. When the path loss exponent is $\eta=4$, the above specialize to

$$
\begin{cases}F_{\rho}(\theta) \simeq e^{-0.854 / \theta} & 0 \leq \theta<0.457 \\ F_{\rho}(\theta) \approx 0.154 & 0.457 \leq \theta<0.5 \\ F_{\rho}(\theta)=1-\frac{4 \sqrt{\theta}-\theta-1}{\pi \theta} & 0.5 \leq \theta<1 \\ F_{\rho}(\theta)=1-\frac{2}{\pi \sqrt{\theta}} & \theta \geq 1 .\end{cases}
$$

An even simpler, slightly less accurate expression is obtained using the exact form only down to $\theta=1$ and resorting to the lower-tail expansion in (15) at that point. This gives

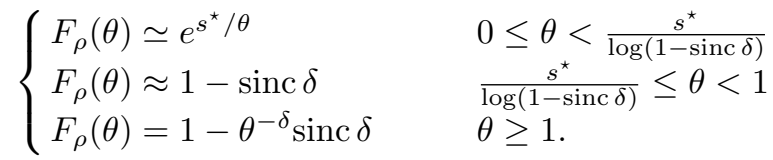

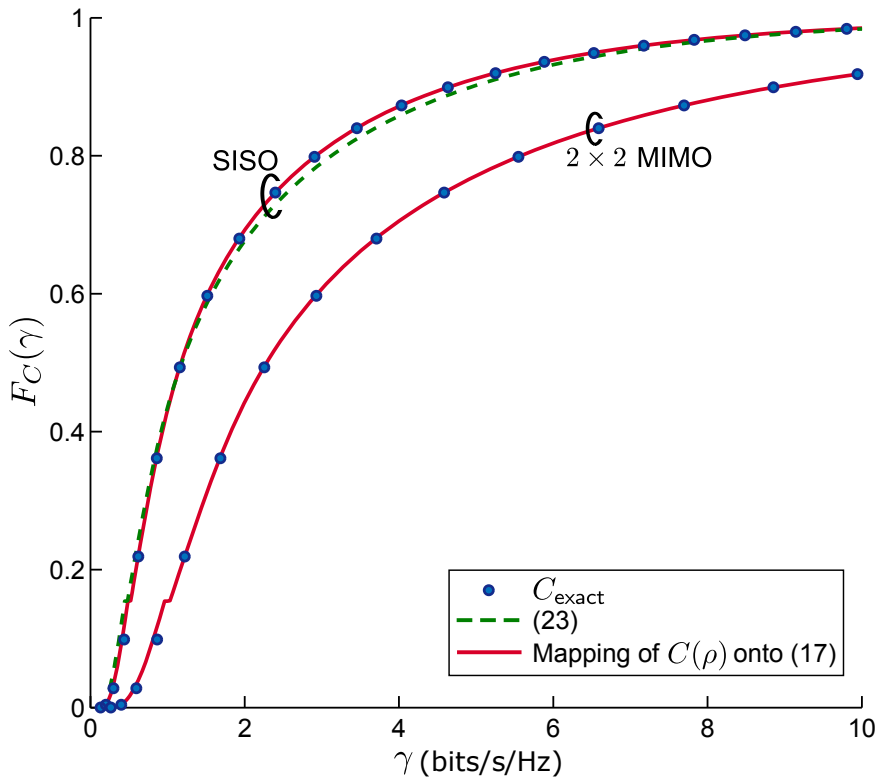

Fig. 2: CDF of ergodic spectral efficiency for $\eta=4$.

\section{Spectral Efficiency: Distribution}

The randomness that $\rho$ acquires once the BS positions are randomized is then inherited by $C$, and the corresponding $\mathrm{CDF}$ provides a complete description of the ergodic spectral efficiency offered by the network over all locations. By mapping the applicable function $C(\rho)$ onto the expressions for $F_{\rho}(\cdot)$ derived in the previous section, $F_{C}(\cdot)$ is readily characterized. In interference-limited conditions, such $F_{C}(\cdot)$ depends only on the path loss exponent, $\eta$.

\section{A. SISO Channels}

In Rayleigh-faded SISO channels, $C(\rho)$ is given by (14). Invoking the invertible approximation [19]

$$
e^{\nu} E_{1}(\nu) \log _{2} e \approx 1.4 \log \left(1+\frac{0.82}{\nu}\right)
$$

we can write $\rho \approx \frac{e^{C / 1.4}-1}{0.82}$ and then, by means of $(17)$,

$$
F_{C}(\gamma) \approx F_{\rho}\left(\frac{e^{\frac{\gamma}{1.4}}-1}{0.82}\right)
$$

whose accuracy is validated next.

Example 3. Consider an interference-limited network with $\eta=4$, where (23) specializes to

$F_{C}(\gamma) \approx \begin{cases}e^{-\frac{0.7}{\exp (\gamma / 1.4)-1}} & 0 \leq \gamma<0.44 \\ 0.154 & 0.44 \leq \gamma<0.48 \\ 1-\frac{4}{\pi} \sqrt{\frac{0.82}{e^{\frac{\gamma}{1.4}}-1}}+\frac{e^{\frac{\gamma}{1.4}}-0.18}{\pi\left(e^{\frac{\gamma}{1.4}}-1\right)} & 0.48 \leq \gamma<0.84 \\ 1-\frac{2}{\pi} \sqrt{\frac{0.82}{e^{\gamma / 1.4}-1}} & \gamma \geq 0.84 .\end{cases}$

Fig. 2 shows (23), as well as the numerical mapping of $C(\rho)-$ without the bypass of its invertible approximation-onto (17), both solutions contrasted against $C_{\text {exact }}$. For the computation of $C_{\text {exact }}$ via Monte-Carlo, here and in all examples involving 
simulations hereafter, PPP-populated BSs (1000 on average) are dropped in a circular area centered at the receiver.

In contrast to these pleasing results, without the model for $z$ propounded in this paper the distribution of the spectral efficiency over the network locations is far more inaccessible. Indeed, the corresponding $C_{\mathrm{ub}}$ is no longer a function of singly $\rho$, whose distribution was established in the previous section; rather, it is a more involved function of $\left\{r_{k}\right\}_{k=0}^{\infty}$ and offers no obvious way of disentangling these dependences. Faced with this obstacle, some authors choose to instead characterize the distribution of

$$
\log _{2}\left(1+\frac{P r_{0}^{-\eta}\left|H_{0}\right|^{2}}{P \sum_{k=1}^{\infty} r_{k}^{-\eta}\left|H_{k}\right|^{2}+N_{0}}\right)
$$

over $\left\{H_{k}\right\}_{k=0}^{\infty}$ as well as $\left\{r_{k}\right\}_{k=0}^{\infty}$. However, the mixing of small- and large-scale variations within this quantity clutters potential observations. Moreover, the generalization to more involved settings, say MIMO, appears arduous or outright hopeless. Indeed, existing stochastic geometry analyses of spectral efficiency featuring MIMO are restricted to beamforming or space-division multiple access, rather than spatial multiplexing [20].

\section{B. MIMO Channels}

Our approach, in contrast, only requires mapping the appropriate $C(\rho)$ onto $F_{\rho}(\cdot)$. Whenever $C(\rho)$ does not lend itself to inversion, even approximately, it is straightforward to perform this mapping numerically.

Example 4. Reconsider Example 3, but now with two transmit and two receive antennas. If $H_{0}$ is replaced by a $2 \times 2$ channel matrix $\boldsymbol{H}_{0}$ having IID Rayleigh-faded entries, then [21]

$$
C_{2 \times 2}^{\text {MIмо }}(\rho)=2 e^{2 / \rho}\left[E_{1}\left(\frac{2}{\rho}\right)+E_{3}\left(\frac{2}{\rho}\right)\right] \log _{2} e
$$

Fig. 2 depicts the numerical mapping of $C_{2 \times 2}^{\text {мImo }}(\rho)$ onto (17), as well as the corresponding $C_{\text {exact }}$.

\section{Spectral Efficiency: Spatial Average}

It is sometimes of interest to condense $F_{C}(\cdot)$ down to a single quantity, with the average being the logical choice. Here again, the approach propounded in this paper proves advantageous. For any setting for which $C(\rho)$ is available, our expressions for $F_{\rho}(\cdot)$ enable computing

$$
\bar{C}=\int_{0}^{\infty} C(\theta) \mathrm{d} F_{\rho}(\theta),
$$

which, in interference-limited conditions, again depends only on the path loss exponent.

\section{A. SISO Channels}

In Rayleigh-faded SISO channels, with (14) and (21) plugged into (26), the integration yields (cf. [4, App. C])

$$
\begin{aligned}
\bar{C} \approx \frac{-s^{\star} \log _{2} e}{1+s^{\star}} & {\left[E_{1}\left(-s^{\star} / D_{\delta}\right)-e^{\left(1+s^{\star}\right) / D_{\delta}} E_{1}\left(1 / D_{\delta}\right)\right] } \\
+ & \frac{\sin (\pi \delta) \log _{2} e}{\pi} G_{2,3}^{2,2}\left(\begin{array}{l|l}
0,1-\delta \\
0,0,-\delta
\end{array}\right)
\end{aligned}
$$

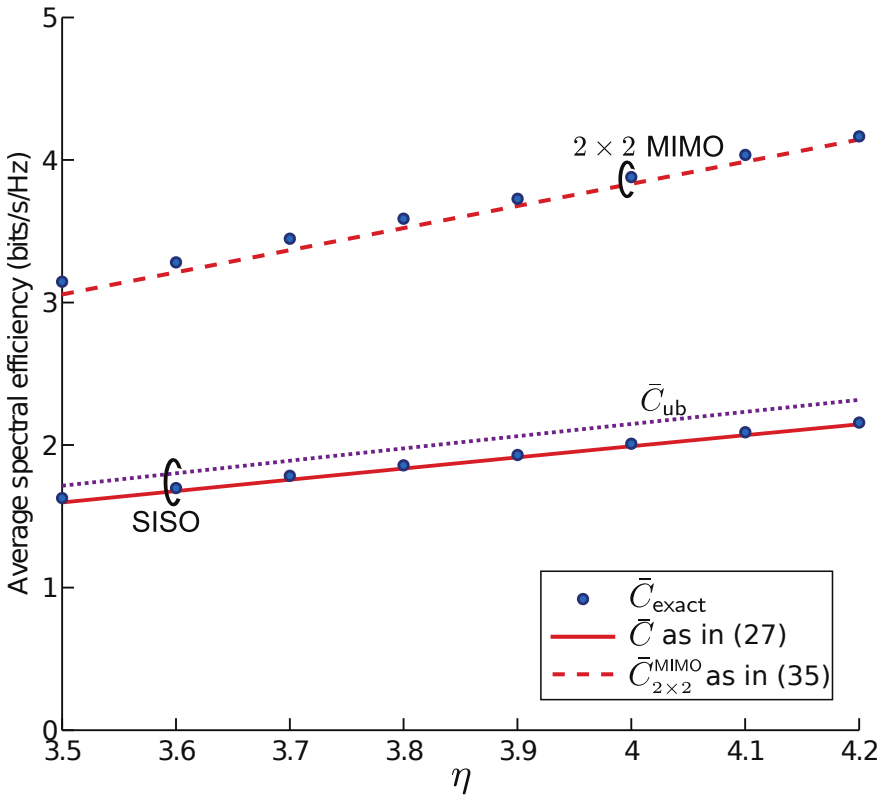

Fig. 3: Spatially averaged ergodic spectral efficiency as a function of $\eta$ for SISO and for $2 \times 2$ MIMO. The $99 \%$ confidence intervals around the MonteCarlo results for $\bar{C}_{\text {exact }}$ range from \pm 0.008 at $\eta=3.5$ to \pm 0.01 at $\eta=4.2$ for SISO and from \pm 0.015 at $\eta=3.5$ to \pm 0.02 at $\eta=4.2$ for MIMO. The SISO upper bound $\bar{C}_{\mathrm{ub}}$ is computed via (31).

where

$$
G_{p, q}^{m, n}\left(\begin{array}{l|l}
z & \begin{array}{l}
a_{1}, \ldots, a_{n}, a_{n+1}, \ldots, a_{p} \\
b_{1}, \ldots, b_{m}, b_{m+1}, \ldots, b_{q}
\end{array}
\end{array}\right)
$$

is the Meijer-G function while $D_{\delta}=s^{\star} / \log (1-\operatorname{sinc} \delta)$. An even more precise, yet also more involved expression for $\bar{C}$, can be obtained using (17) in lieu of (21).

Example 5. Fig. 3 compares (27) against $\bar{C}_{\text {exact }}$, which is the Monte-Carlo average of $C_{\text {exact }}$, for $3.5 \leq \eta \leq 4.2$. For $\eta=4$ in particular,

$$
\begin{aligned}
& \bar{C} \approx 0.187+\frac{\log _{2} e}{\pi} G_{2,3}^{2,2}\left(\begin{array}{l|l}
1 & \begin{array}{l}
0,1 / 2 \\
0,0,-1 / 2
\end{array}
\end{array}\right) \\
& =1.99
\end{aligned}
$$

while $\bar{C}_{\text {exact }}$ is 2.01 .

It is worthwhile to contrast (27) with its counterpart obtained without the model for $z$ propounded in this paper, namely the average of $C_{\mathrm{ub}}$ given by [1]

$$
\bar{C}_{\mathrm{ub}}=\int_{0}^{\infty} \frac{\log _{2} e}{1+\left(e^{t}-1\right)^{\delta} \int_{\left(e^{t}-1\right)^{-\delta}}^{\infty} \frac{1}{1+x^{1 / \delta}} \mathrm{d} x} \mathrm{~d} t .
$$

Example 6. Included in Fig. 3, next to its SISO counterparts $\bar{C}$ and $\bar{C}_{\text {exact }}$, is also $\bar{C}_{\mathrm{ub}}$.

Besides being further from $C_{\text {exact }}$ than our solution $\bar{C}$, and requiring a double integration, the expression for $\bar{C}_{\mathrm{ub}}$ does not offer a viable path to MIMO generalization. With our approach, in contrast, the analysis of the spatial average becomes feasible also with MIMO. 


\section{B. MIMO Channels}

For $2 \times 2 \mathrm{MIMO}$, the integration of $C_{2 \times 2}^{\mathrm{MIMO}}(\rho)$ as given in (25) over $F_{\rho}(\cdot)$ as given in (21) returns a rather lengthy expression that is deferred to [4]. An alternative expression not involving the Meijer-G function can be obtained using

$$
E_{3}(x) \approx \frac{e^{-3 x / 2}}{2}
$$

and

$$
2 e^{\frac{2}{\rho}} E_{1}\left(\frac{2}{\rho}\right) \log _{2} e \approx 2.8 \log (1+0.41 \rho)
$$

to simplify $C_{2 \times 2}^{\text {MIMO }}(\rho)$ in (25) into

$$
C_{2 \times 2}^{\mathrm{MIMO}}(\rho) \approx 2.8 \log (1+0.41 \rho)+e^{-1 / \rho} \log _{2} e .
$$

Using this form in place of (25) in the integration gives (cf. [4, App. C])

$$
\begin{aligned}
& \bar{C}_{2 \times 2}^{\text {Miмо }} \approx \frac{s^{\star} \log _{2} e}{\left(s^{\star}-1\right) e^{\frac{s^{\star}-1}{D \delta}}}+\delta \operatorname{sinc}(\delta) \bar{\Gamma}(\delta, 1) \log _{2} e \\
& +2.8\left[{ }_{2} F_{1}(1, \delta, 1+\delta,-2.44)+\delta \log (1.41)\right] \frac{\operatorname{sinc}(\delta)}{\delta} \\
& +2.8\left[\frac{E_{1}\left(-s^{\star}\left(0.41+1 / D_{\delta}\right)\right)}{e^{0.41 s^{\star}}}-E_{1}\left(-s^{\star} / D_{\delta}\right)\right. \\
& \left.+e^{s^{\star} / D_{\delta}} \log \left(1+0.41 D_{\delta}\right)\right] \text {. }
\end{aligned}
$$

Example 7. Fig. 3 compares (35) against $\bar{C}_{\text {exact. }}$ For $\eta=4$ in particular, (35) returns

$$
\begin{aligned}
\bar{C}_{2 \times 2}^{\text {мाмо }} \approx & 0.26+\frac{\log _{2} e}{\sqrt{\pi}} \operatorname{erf}(1) \\
& +\frac{5.6}{\pi}[\sqrt{0.41}(\pi-2 \arctan (\sqrt{0.41}))+\log 1.41] \\
= & 3.84
\end{aligned}
$$

while the Monte-Carlo average of its $C_{\text {exact }}$ counterpart is 3.87 .

Combining Examples 5 and 7, two-antenna single-user MIMO is seen from our analysis to provide a $93 \%$ increase in the spectral efficiency of an entire interference-limited network, a result that would classically have entailed very extensive simulations.

For more than two antennas, the reader is referred to [4].

\section{SUMmary}

By decoupling small- and large-scale channel features and abstracting the former via local ergodicity, the stochastic geometry analysis of wireless networks can focus crisply on the large-scale properties. Jointly with a Gaussian model for the aggregate interference that recognizes that the fading of each term therein is unknown, this enables circumventing analytical roadblocks and deriving expressions that are simpler, more accurate, and more open to generalization, readily accommodating aspects such as MIMO. The obtained spectral efficiencies lower-bound the exact values with a degree of accuracy that justifies writing $C \lesssim C_{\text {exact }}$.
The approach propounded in this paper is extensible to settings where noise is nonnegligible, and the accuracy of the results could then only improve even further since our interference model is an exact match for Gaussian noise devoid of fading.

\section{REFERENCES}

[1] J. G. Andrews, F. Baccelli, and R. K. Ganti, "A tractable approach to coverage and rate in cellular networks," IEEE Trans. Commun., vol. 59, no. 11 , pp. 3122-3134, Nov. 2011.

[2] B. Błaszczyszyn, M. K. Karray, and H. P. Keeler, "Wireless networks appear Poissonian due to strong shadowing," IEEE Trans. Wireless Commun., vol. 14, no. 8, pp. 4379-4390, Aug. 2015.

[3] H. P. Keeler, N. Ross, and A. Xia, "When do wireless network signals appear Poisson?" 2014. [Online]. Available: http://arxiv.org/abs/1411. 3757

[4] G. George, R. K. Mungara, A. Lozano, and M. Haenggi, "Ergodic spectral efficiency in MIMO cellular networks," IEEE Trans. Wireless Commun., to appear, 2017.

[5] A. Lozano and N. Jindal, "Transmit diversity vs. spatial multiplexing in modern MIMO systems," IEEE Trans. Wireless Commun., vol. 9, no. 1, pp. 186-197, Jan. 2010.

[6] _ "Are yesterday's information-theoretic fading models and performance metrics adequate for the analysis of today's wireless systems?" IEEE Commun. Mag., vol. 50, no. 11, pp. 210-217, Nov. 2012.

[7] A. Lozano, "Interplay of spectral efficiency, power and Doppler spectrum for reference-signal-assisted wireless communication," IEEE Trans. Wireless Commun., vol. 7, no. 12, pp. 5020-5029, Dec. 2008.

[8] A. Lapidoth and S. Shamai, "Fading channels: how perfect need "perfect side information" be?" IEEE Trans. on Inform. Theory, vol. 48, no. 5, pp. 1118-1134, 2002

[9] S. Ak, H. Inaltekin, and H. V. Poor, "Gaussian approximation for the downlink interference in heterogeneous cellular networks," 2016. [Online]. Available: http://arxiv.org/abs/1601.06023

[10] S. N. Diggavi and T. M. Cover, "The worst additive noise under a covariance constraint," IEEE Trans. on Inform. Theory, vol. 47, no. 7, pp. 3072-3081, 2001.

[11] M. Haenggi, "On distances in uniformly random networks," IEEE Trans. Inform. Theory, vol. 51, pp. 3584-3586, Oct. 2005.

[12] M. Zorzi, "On the analytical computation of the interference statistics with applications to the performance evaluation of mobile radio systems," IEEE Trans. Commun., vol. 45, no. 1, pp. 103-109, Jan. 1997.

[13] H. S. Dhillon, R. K. Ganti, F. Baccelli, and J. G. Andrews, "Modeling and analysis of $K$-tier downlink heterogeneous cellular networks," IEEE J. Select. Areas Commun., vol. 30, no. 3, pp. 550-560, Apr. 2012.

[14] B. Błaszczyszyn, M. K. Karray, and H. P. Keeler, "Using Poisson processes to model lattice cellular networks," in Proc. IEEE INFOCOM, Apr. 2013, pp. 773-781.

[15] S. Mukherjee, "Downlink SINR distribution in a heterogeneous cellular wireless network with max-SINR connectivity," in Proc. Annual Allerton Conf. Commun., Cont., Computing, Sep. 2011, pp. 1649-1656.

[16] B. Błaszczyszyn and H. P. Keeler, "Studying the SINR process of the typical user in Poisson networks using its factorial moment measures," IEEE Trans. Inform. Theory, vol. 61, no. 12, pp. 6774-6794, Dec 2015.

[17] B. Błaszczyszyn and M. K. Karray, "Spatial distribution of the SINR in Poisson cellular networks with sector antennas," IEEE Trans. Wireless Commun., vol. 15, pp. 581-593, Jan. 2016.

[18] R. K. Ganti and M. Haenggi, "SIR asymptotics in Poisson cellular networks without fading and with partial fading," in Proc. IEEE Int. Conf. Commun., May 2016.

[19] S. Catreux, P. F. Driessen, and L. J. Greenstein, "Data throughputs using multiple-input multiple-output (MIMO) techniques in a noise-limited cellular environment," IEEE Trans. Wireless Commun., vol. 1, no. 2, pp. 226-235, Apr. 2002.

[20] A. K. Gupta, H. S. Dhillon, S. Vishwanath, and J. G. Andrews, "Downlink multi-antenna heterogeneous cellular network with load balancing," IEEE Trans. Commun., vol. 62, pp. 4052-4067, Nov. 2014.

[21] H. Shin and J. H. Lee, "Capacity of multiple-antenna fading channels: spatial fading correlation, double scattering, and keyhole," IEEE Trans. Inform. Theory, vol. 49, no. 10, pp. 2636-2647, Oct. 2003. 